\title{
A Double-Blind, Randomized, Placebo-Controlled, Two-Dose Comparative Study of Botulinum Toxin Type A for Treating Glabellar Lines in Japanese Subjects: What If Sample Size and Statistical Tests Mattered?
}

\author{
Alberto Mangano · Andrea Albertin · \\ Luca La Colla
}

Published online: 20 June 2009

(C) Springer Science+Business Media, LLC and International Society of Aesthetic Plastic Surgery 2009

We read with interest the article published by Harii and Kawashima [1]. While the authors are to be commended for investigating the effect of different doses of botulinum toxin type A (BTX-A) in Japanese subjects, there are several issues with both the design of the study and the statistical analysis used. With respect to the study design, despite the statement from the authors that it was a prospective, randomized, double-blind, placebo-controlled study, it does not appear to be so. There was no hypothesis to test; it appears to be a pilot study. Even if the authors wanted to test the hypothesis that halving the dose of BTXA (from 20 to $10 \mathrm{U}$ ) would not result in a significant decrease in a patient's response rate, the sample size calculation is still missing. In fact, in order for a particular finding to be claimed as significant (or not), the study must have enough power. The authors do not state anything about the expected change in response rate values and therefore it is almost impossible to perform a prestudy sample size calculation. However, it is possible to perform a post-hoc sample size calculation or power analysis on the statistical tests they have used from their data. In particular, considering a rough response rate of $90 \%$ in the $20-\mathrm{U}$ group, a $10 \%$ clinically significant difference in response

A. Mangano

Vita-Salute San Raffaele University School of

Medicine - IRCCS San Raffaele, Milan, Italy

\section{A. Albertin}

Department of Anesthesiology, IRCCS Multimedica,

Sesto San Giovanni, Milan, Italy

L. La Colla $(\bowtie)$

Department of Anesthesiology, Vita-Salute San Raffaele

University School of Medicine - IRCCS San Raffaele,

Via Olgettina 60, 20132 Milan, Italy

e-mail:1.lacolla@studenti.hsr.it rate, and the Bonferroni's correction for multiple comparisons, more than 160 patients per group would have been required (considering the "standard" $\alpha$ error of 0.05 and a power of 0.8 ). Moreover, the randomization method is not clearly explained.

Another critical point is the statistical analysis. The authors state that the $t$ test and ANOVA were used for continuous variables. This may not be the correct way to go. Are all the variables normally distributed? If not, nonparametric tests should be used. Moreover, the authors state they used a nonparametric test on categorical variables. Why? Could a categorical variable be non-normally distributed?

While this study is interesting for plastic and cosmetic surgeons and dermatologists, appropriate statistical analysis of data from trials is fundamental to draw adequate conclusions. We recommend that all authors perform the right sample size calculation before beginning any type of prospective, randomized, controlled trial and use the correct statistical tests afterward. This is the only way to convince readers that what they claim to be significant is really so. We hope our suggestions will be useful to other authors who will be doing similar studies in the future.

\section{Reference}

1. Harii K, Kawashima M (2008) A double-blind, randomized, placebo-controlled, two-dose comparative study of botulinum toxin type A for treating glabellar lines in Japanese subjects. Aesthetic Plast Surg 32:724-730 